\title{
MONITORAMENTO ESPAÇO-TEMPORAL DAS ÁREAS DE ALTO RISCO DE COVID-19 NOS MUNICÍPIOS DO BRASIL
}

\section{SPACE-TIME MONITORING OF COVID-19 HIGH-RISK AREAS IN BRAZILIAN MUNICIPALITIES}

\author{
Ligia Vizeu Barrozo \\ Professora associada do Departamento de Geografia e do \\ Programa de Pós Graduação em Geografia Física da Universidade de São Paulo \\ Pesquisadora do Grupo de Estudos Espaço Urbano e Saúde do Instituto de Estudos Avançados da Universidade \\ de São Paulo, SP, Brasil \\ lija@usp.br \\ Mirela Barros Serafim \\ Doutoranda pelo Programa de Pós Graduação em Geografia Física da Universidade de São Paulo \\ Pesquisadora do Grupo de Estudos Espaço Urbano e Saúde do Instituto de Estudos Avançados da Universidade \\ de São Paulo, SP, Brasil \\ mirelaserafim@usp.br \\ Sara Lopes de Moraes \\ Doutoranda pelo Programa de Pós Graduação em Geografia Física da Universidade de São Paulo \\ Pesquisadora do Grupo de Estudos Espaço Urbano e Saúde do Instituto de Estudos Avançados da Universidade \\ de São Paulo, SP, Brasil \\ sara.moraes@usp.br \\ Giselle Mansur \\ Doutoranda pelo Programa de Pós Graduação em Geografia Física da Universidade de São Paulo \\ Pesquisadora do Grupo de Estudos Espaço Urbano e Saúde do Instituto de Estudos Avançados da Universidade \\ de São Paulo, SP, Brasil \\ gisellemansur@usp.br
}

\begin{abstract}
RESUMO
Em 11 de março de 2020 a doença COVID-19 foi declarada uma pandemia. No Brasil o primeiro registro oficial da doença foi em 25 de fevereiro de 2020 , contabilizando até o dia 01 de Maio do mesmo ano um total de 90.982 casos e 6.367 óbitos. Utilizando-se do software SaTScan ${ }^{\mathrm{TM}}$, a partir de ferramentas de estatística espacial e espaço-temporal, é possível elaborar análises robustas de varredura retrospectiva e prospectiva para identificação da ocorrência e acompanhar o espalhamento da doença no espaço e no tempo. Nesse contexto foi criado o projeto MONITORA-CLUSTERS, cujo objetivo é verificar e acompanhar agrupamentos de alto risco dos casos confirmados e óbitos por COVID-19. Ao longo do período estudado os resultados mostraram a formação de agrupamentos de alto risco relativo significativos nas modelagens espaciais e espaço-temporais. A fim de identificar agrupamentos ativos de casos e de óbitos, a estatística de varredura espaçotemporal prospectiva mostra onde e quando tiveram início tais agrupamentos, apontando locais críticos para medidas de controle. Percebeu-se em ambas as abordagens que todas as macrorregiões brasileiras apresentam aglomerados com excesso de casos registrados e/ou de óbitos. Essa poderosa ferramenta de vigilância epidemiológica tem potencial para colaborar no auxílio de tomadas de decisão referentes à pandemia no país.
\end{abstract}

Palavras-chave: COVID-19. SaTScan. Agrupamentos espaciais. Agrupamentos espaçotemporais. análise espacial.

\section{ABSTRACT}

On March 11th, 2020, the COVID-19 disease has declared a pandemic. In Brazil, the first official record of the disease was on February 25, 2020, a total of 90,982 cases and 6,367

Recebido em: 08/05/2020

Aceito para publicação em: 21/05/2020. 
deaths were counted until May 1st of the same year. Using the spatial and space-time statistical tools of the SaTScan ${ }^{\mathrm{TM}}$ software, it is possible to develop a robust retrospective and prospective scanning analysis to identify and monitor the occurrence of the spread of the disease. In this context, the MONITORA-CLUSTERS project was created, which aims to verify and monitor high-risk clusters of confirmed cases and deaths by COVID-19 in Brazil. Throughout the study period, the results showed

spatial and space-time significant clusters. To identify active clusters of cases and deaths, the prospective spatio-temporal analysis shows where and when such clusters began, pointing out critical locations for control measures. It was noticed in both approaches that all Brazilian macro-regions present clusters with an excess of registered cases and/or deaths. This powerful epidemiological tool has the potential to collaborate in assisting pandemic decision-making in the country.

Keywords: COVID-19. SaTScan. Spatial clusters. Space-time clusters. Spatial analysis.

\section{INTRODUÇÃO}

A doença infecciosa COVID-19 causada pelo novo coronavírus da síndrome respiratória aguda grave 2 (SARS-CoV-2) tornou-se um grande problema de saúde pública ao redor do mundo. O primeiro surto da doença foi comunicado pela China, com identificação de pacientes com pneumonia de etiologia desconhecida, na cidade de Wuhan, em dezembro de 2019 (HUANG et al., 2020; LU; STRATTON; TANG, 2020). Em um mês, durante o Ano Novo Lunar, o vírus se espalhou rapidamente na China, apresentando um aumento de número de casos quase exponencial (ADHIKARI et al., 2020; KANG et al., 2020). Posteriormente, a doença atingiu diversos países da Ásia, Europa, América e África, sendo declarada uma pandemia pela Organização Mundial da Saúde (OMS) no dia 11 de março, quando os casos da doença já haviam sido notificados em 114 países (LAKHANI, 2020). Comparado com os vírus SARS-CoV e MERS-CoV que causaram os surtos de 2002/2003 e de 2012/2014, respectivamente, o espalhamento da COVID-19 foi muito mais rápido. Em apenas 48 dias havia 1.000 pessoas infectadas por COVID-19, enquanto o mesmo número de infecções causadas pelo SARS-CoV foi registrado cerca de quatro meses após o início da epidemia (KAMEL BOULOS e GERAGHTY, 2020).

A COVID-19 é uma doença predominantemente respiratória, podendo acometer outros sistemas, como cutâneo, neurológico e hematológico. As mais prováveis vias de transmissão são por gotículas, contato físico e aerossóis (LIU et al., 2020). Seu período médio de incubação tem sido estimado em 7 dias, variando de 2 a 14 (ADHIKARI et al., 2020). As populações s' envolvem idosos, pessoas com função imune comprometida, aquelas com comorbidades crônicas, as que usam imunossupressores por longo tempo e as com histórico cirúrgico antes da internação. Até o presente momento não há tratamento antiviral específico e tampouco vacina, de modo que a mais efetiva forma de prevenção consiste em evitar a exposição ao vírus (ADHIKARI et al., 2020). As principais medidas de saúde pública para a contenção ou desaceleração da epidemia incluem distanciamento social, isolamento dos casos confirmados, identificação e acompanhamento dos contatos, desinfecção ambiental e uso de equipamentos protetivos individuais (HAMID et al., 2020).

A COVID-19 é uma doença predominantemente respiratória, podendo acometer outros sistemas, como tegumentar, neurológico e hematológico. As mais prováveis vias de transmissão são por gotículas e contato físico, sendo ainda controverso que possa ocorrer através de aerossóis (LIU et al., 2020). Seu período médio de incubação tem sido estimado em 7 dias, variando de 2 a 14 (ADHIKARI et al., 2020). As populações de maior risco incluem - idosos; pessoas com função imune comprometida; aquelas com certas comorbidades crônicas, particularmente diabetes, hipertensão e obesidade; as que usam imunossupressores por longo tempo; e aquelas com histórico cirúrgico antes da internação, São, porém, também suscetíveis, indivíduos sem fatores de risco. A maioria dos infectados são assintomáticos ou desenvolvem formas leves da doença. Até o presente momento não há tratamento antiviral específico e tampouco vacina, de modo que a mais efetiva forma de prevenção consiste em evitar a exposição ao vírus (ADHIKARI et al., 2020). As principais medidas de saúde pública para a contenção ou desaceleração da epidemia incluem distanciamento social, isolamento dos casos confirmados, identificação e acompanhamento dos contatos, desinfecção ambiental e uso de equipamentos protetivos individuais (HAMID et al., 2020). 
As contribuições científicas mais relevantes sobre o espalhamento da pandemia envolvem principalmente modelos epidemiológicos determinísticos de evolução temporal com finalidades preditivas, enquanto o espaço tem sido tratado quase marginalmente na literatura internacional (GIULIANI et al., 2020). Há ainda poucas dezenas de exemplos de estudos que adotaram abordagens espaciais para analisar a evolução da pandemia de COVID-19 no mundo, a maioria retrospectiva (KANG et al., 2020; YANG et al., 2020). Desjardins et al. (2020) foram os primeiros a publicar uma análise espaço-temporal prospectiva para a detecção de agrupamentos ativos dos casos nos Estados Unidos. A ferramenta estatística de varredura espacial retrospectiva e de varredura espaço-temporal prospectiva para a detecção de agrupamentos ativos (KULLDORFF e Information Management Services, 2018) tem sido usada em diferentes desfechos de saúde, incluindo surtos de doenças infecciosas, como as causadas pelos vírus da gripe e da gripe de origem suína (WILSON et al., 2010).

Segundo Kang et al. (2020), que estudaram os vinte primeiros dias de disseminação da infecção na China continental, a análise espaço-temporal do início da epidemia é crítica para a predição de surtos locais, sendo de fundamental importância para orientar políticas públicas e prevenir a transmissão futura. Metodologias de análise espacial também têm sido aplicadas com outras finalidades, por exemplo no mapeamento de hotspots de casos para a identificação de áreas para a aplicação de testes de rápido diagnóstico aplicados no local de atendimento (KOST, 2020), na identificação de áreas prioritárias para a alocação de recursos de saúde em regiões da Austrália (LAKHANI, 2020a; LAKHANI, 2020b) e na aplicação de modelos espaciais de dados em painel para a determinação dos efeitos espaciais associados à distribuição da COVID-19 (GULIYEV, 2020).

Os Sistemas de Informações Geográficas (SIG), como importantes instrumentos de análise espaçotemporal, são requeridos no monitoramento do contágio de doenças. A alta velocidade de espalhamento da doença gera a necessidade de as informações epidêmicas serem disponibilizadas ainda mais rápido (KAMEL BOULOS e GERAGHTY, 2020). No contexto da pandemia, a utilização de SIG e de tecnologias de big data têm possibilitado a agregação de amplos bancos de dados, de inúmeras fontes, bem como a visualização de tais informações, atualizadas em período de tempo hábil à tomada de medidas de prevenção à infecção. Os dashboards de mapeamentos desenvolvidos pela Johns Hopkins University's Center for Systems Science and Engineering (JHU CSSE, 2020), pela World Health Organization (WHO, 2020) e pela Early Alert Inc. (EARLY ALERT, 2020) são exemplos de tecnologias SIG baseadas em web amplamente acessadas tanto pelo público quanto por profissionais da área da saúde e responsáveis por políticas públicas (KAMEL BOULOS e GERAGHTY, 2020).

O monitoramento da ocorrência de COVID-19 nos municípios brasileiros, proposto no âmbito do Projeto MONITORA-CLUSTERS, tem como finalidade a identificação dos municípios e dos agrupamentos de municípios com excesso de casos confirmados e de óbitos para COVID-19. Para tal, são verificados os casos esperados em relação ao tamanho de suas populações e à ocorrência da doença em todo o território nacional através da utilização de análise espacial retrospectiva e espaço-temporal prospectiva. A análise prospectiva permite verificar a data de início dos agrupamentos de alto risco de COVID-19 ainda ativos até a data do último caso ou óbito do banco de dados.

Dessa forma, o objetivo da presente pesquisa é monitorar os agrupamentos espaciais e espaçotemporais ativos de alto risco no território nacional durante o curso da epidemia no Brasil, evidenciando as localidades que apresentam excesso de casos e óbitos para a COVID-19. Trata-se de potente instrumento no auxílio das tomadas de decisão relacionadas à orientação da prática de distanciamento social, aumento do número de testes para diagnóstico, alocação de recursos e de serviços de saúde, por exemplo, a eventual designação de ambulâncias para municípios sem serviço de saúde de alta complexidade, entre outras medidas urgentes frente à situação epidêmica.

\section{DADOS E MÉTODOS}

\section{Dados}

O monitoramento dos agrupamentos é composto por dois tipos de análise de varredura, uma espacial retrospectiva e outra espaço-temporal prospectiva. As abordagens envolvem estatística espacial a partir (i) das coordenadas geográficas das sedes dos municípios brasileiros, referenciados no sistema geodésico SIRGAS 2000; (ii) da população estimada pelo Instituto Brasileiro de Geografia e Estatística (IBGE), adotando como data de referência 1 de julho de 2019; (iii) do número de casos 
diagnosticados e de óbitos para COVID-19, disponibilizados pelas Secretarias Estaduais de Saúde e compiladas no endereço eletrônico do projeto Brasil.IO (https://brasil.io/dataset/covid19/caso/).

\section{Métodos}

\section{Análises de varredura espacial retrospectiva e espaço-temporal prospectiva}

As análises de varredura espacial e espaço-temporal (KULLDORFF e Information Management Services, 2018) são realizadas no software SaTScan ${ }^{\mathrm{TM}}$, com base no modelo discreto de Poisson e com escala temporal diária.

Para compreender as análises espacial e espaço-temporal, deve-se recorrer às imagens de um círculo e de um cilindro. $\mathrm{Na}$ análise espacial, o programa coloca o centro do raio do círculo na sede do município e faz uma varredura, abrindo o raio do círculo até encontrar um risco maior da ocorrência de casos e de óbitos dentro do círculo quando comparado ao risco fora do círculo. $O$ nosso monitoramento permite que este raio atinja o máximo de $10 \%$ da população do Brasil (ou cerca de 21 milhões de habitantes). O círculo máximo permitido pelo programa seria de até $50 \%$ da população em risco, o que poderia incluir metade da população brasileira e não contribuir para a identificação dos agrupamentos menores. O valor da janela espacial de $10 \%$ baseou-se no valor indicado nos resultados do Gini index do SaTScan ${ }^{\mathrm{TM}}$, disponibilizado após uma primeira rodada da análise utilizando o valor padrão da janela espacial de $50 \%$ da população em risco. Quando a análise encontra um círculo com risco relativo maior do que o de fora do círculo, são processadas réplicas do tipo Monte Carlo para o cálculo do valor de $\mathrm{p}$ (considerado no estudo como significativo quando for menor do que 0,05 ). O programa foi ajustado para rodar o número máximo de 999 réplicas. $\mathrm{Na}$ sequência do primeiro agrupamento encontrado, o programa passa a varrer outros municípios para encontrar prováveis agrupamentos na área de estudo, reproduzindo o procedimento do primeiro. A varredura é feita de forma a considerar todas as sedes de municípios do Brasil, com milhares de círculos testados. Como resultado da varredura tem-se o Risco Relativo (RR) calculado para cada município do Brasil. O RR menor do que 1 indica que ocorreram menos casos ou óbitos do que o esperado para a população em risco. No caso de uma doença infecciosa como a COVID-19, o RR menor do que 1 deve ser visto com muita cautela, porque mesmo que ainda não configure um agrupamento alto, a presença de casos no município requer medidas para evitar o espalhamento. $O$ RR maior do que 1 significa que ocorreram mais casos ou óbitos do que o esperado para aquela população e que pode constituir um agrupamento alto se somado aos seus vizinhos tiver um RR mais elevado estatisticamente do que os municípios que ficaram fora do círculo.

A análise espaço-temporal prospectiva segue exatamente o princípio do círculo na base do cilindro, mas acrescenta a dimensão temporal na sua geratriz (altura). Na análise prospectiva, a varredura tem como objetivo identificar espacialmente quais áreas apresentam risco alto em comparação aos municípios de fora do círculo e, temporalmente, quando tiveram início os agrupamentos significativos que estão ativos até a data do último caso ou óbito analisado. Os parâmetros inseridos foram aqueles da análise espacial, acrescidos dos referenciais de tempo de duração mínima e máxima dos agrupamentos, nesse caso de 2 a 7 dias, permitindo identificar agrupamentos temporais que incluem até o máximo de $50 \%$ do período analisado. A abordagem prospectiva utilizada para a análise de varredura espaço-temporal possui a vantagem, em relação a abordagens retrospectivas, de indicar os agrupamentos estatisticamente significativos emergentes no período, desde que ainda presentes na data final do último caso ou óbito do banco de dados. Desse modo, desconsidera os agrupamentos que possam ter existido anteriormente mas que deixaram de ser uma ameaça à saúde pública (KULLDORFF, 2001). Esse tipo de análise é utilizada para monitoramento em "tempo-real". As análises espaciais retrospectiva e espaço-temporal prospectiva são, assim, complementares.

\section{Representação cartográfica online}

Os resultados obtidos das análises de varredura espacial e espaço-temporal em formato shapefile dos círculos (aglomerados) e pontos (municípios dentro dos aglomerados) foram tratados no ArcMap 10.7 para a exclusão dos círculos com valores de $p>0,05$ e para adicionar os nomes dos municípios e unidades federativas ao arquivo de pontos por meio da função "juntar" tabela de dados (join). $\mathrm{Na}$ sequência, estas camadas foram adicionadas a um projeto no ArcGIS online (arcgis.com). Depois de formatar o layout para a visualização dos mapas, estes foram compartilhados de forma a serem anexados no site na web - espacourbanoesaude.iea.usp.br/monitora-clusters. Este site do grupo de estudos Espaço Urbano e Saúde, do Instituto de Estudos Avançados da USP está hospedando os resultados do projeto de monitoramento da COVID-19 (MONITORA CLUSTERS Brasil). 


\section{RESULTADOS E DISCUSSÃO}

No período de estudo ( 25 de fevereiro a 1 de maio de 2020), ocorreram 90.982 casos notificados e 6.367 óbitos por COVID-19 que tiveram referência do município de residência e que puderam ser analisados. A representação dos dados das análises por meio dos mapas permite a identificação dos agrupamentos espaciais ocorridos no período, e dos agrupamentos espaço-temporais de alto risco significativos que estavam ativos até a data dos dados, no dia 1 de maio.

Os mapas da Figura 1 apresentam os resultados da análise espacial para os casos (Fig. 1A) e óbitos (Fig. 1B) registrados nos municípios brasileiros. Os círculos em vermelho indicam os agrupamentos espaciais de alto risco, ou seja, áreas com excesso de casos ou óbitos ocorridos em relação ao tamanho das suas populações.

Figura 1 - Agrupamentos espaciais de casos (A) e de óbitos (B) no período de 25/02/2020 a $01 / 05 / 2020$

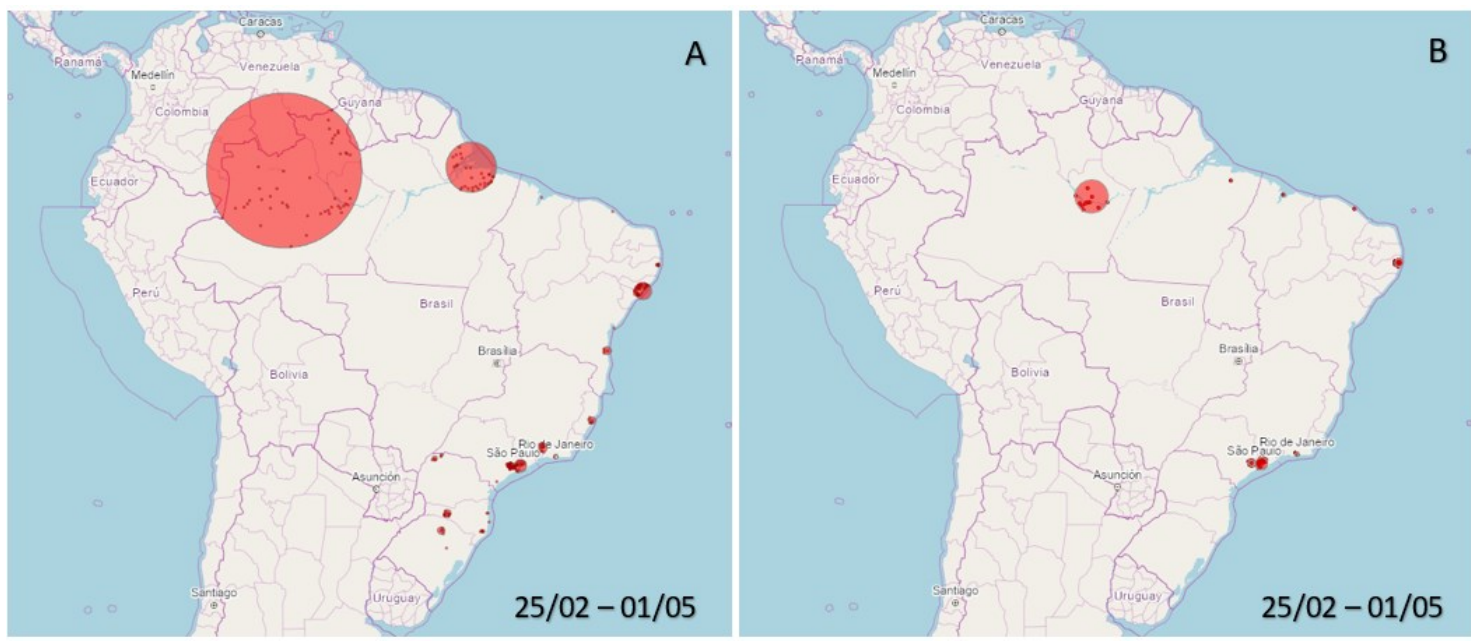

Fonte: https://espacourbanoesaude.iea.usp.br/monitora-clusters.

Nota-se diversos agrupamentos espaciais de alto risco de casos registrados por COVID-19 em todas as macrorregiões brasileiras (Fig. 1A). Foram encontrados 24 agrupamentos significativos. $O$ agrupamento com o maior número corresponde ao centrado em Taboão da Serra/SP e inclui outros 17 municípios, totalizando 23.386 casos. No segundo ocorreram 19.313 e está centrado em São Caetano do Sul/SP, incluindo o Município de São Paulo. O de Fortaleza/CE compreende apenas este município, com 6.082 casos.

Em relação aos óbitos, observa-se a ocorrência de 10 agrupamentos espaciais de alto risco significativos (Fig. 1B), presentes nos estados de São Paulo, Rio de Janeiro, Pernambuco, Ceará, Maranhão e Amazonas. O maior número de óbitos ocorreu no agrupamento que tem como centro o Município de Osasco/SP e inclui 15 outros municípios, inclusive São Paulo. Neste agrupamento ocorreram 1.864 óbitos. O segundo, formado por São Caetano do Sul/SP e São Paulo, teve 1.620 óbitos. O terceiro maior é o de Manaus/AM, com 424 óbitos até a data final desse estudo.

É importante ressaltar que, como as análises usam um círculo ou cilindro, alguns municípios dentro da área de alto risco podem apresentar risco relativo abaixo de 1 (menos casos do que o esperado), mas como se encontram dentro do círculo, também compõem o agrupamento. Nesse sentido, localizar-se dentro do raio de uma área que apresenta excesso de casos indica a necessidade de atenção e eventuais medidas de restrição de contato para com o seu entorno. Conforme os resultados encontrados por Kang et al. (2020) no estudo na China, a COVID-19 apresenta forte autocorrelação espacial, havendo aumento do número de casos nas áreas adjacentes ao foco.

Os resultados da análise espaço-temporal prospectiva representados nas figuras 2 e 3 permitem identificar os locais dos agrupamentos de excesso de casos e óbitos ativos até a data dos dados. A sequência de imagens fornece as informações de data de início dos agrupamentos e da sua localização, possibilitando o monitoramento das áreas de risco que apresentam ameaça à saúde DOl:http://dx.doi.org/10.14393/Hygeia0054547 $\quad$ Hygeia Edição Especial: Covid-19, Jun./2020 p.417 - 425, pág. 421 
pública. Este tipo de informação pode ser usada como subsídio para tomadas de decisão, entre elas a manutenção de distanciamento social, identificação dos locais onde devem ser aplicados mais testes diagnósticos e a necessidade de reforçar todo o sistema de saúde. Essas análises estatísticas pretendem contribuir com uma vigilância em tempo-real de ocorrência dos agrupamentos de casos e de óbitos. "Tempo-real" aqui deve ser entendido como o tempo de ocorrência dos agrupamentos espaciais ativos até a data do último caso ou óbito do banco de dados.

A Figura 2 apresenta os mapas de agrupamentos espaço-temporais ativos de casos ao longo do tempo. Até o dia 01 de maio estavam ativos 11 agrupamentos. O agrupamento com o maior número de casos tem como centro o município de São Bernardo do Campo/SP e está ativo desde 31 de março, com 23.971 casos desde esta data. O segundo agrupamento tem como centro o município de Pedra Grande/RN e inclui 484 outros municípios, onde ocorreram 13.101 casos. Este agrupamento está ativo desde o dia 12 de abril. O terceiro agrupamento ativo tem seu centro em Oiapoque/AP e inclui 372 outros municípios, com 11.294 casos e ativo desde 14 de abril.

Figura 2 - Agrupamentos espaço-temporais de excesso de casos registrados de COVID-19 ativos até o dia 01/05/20.

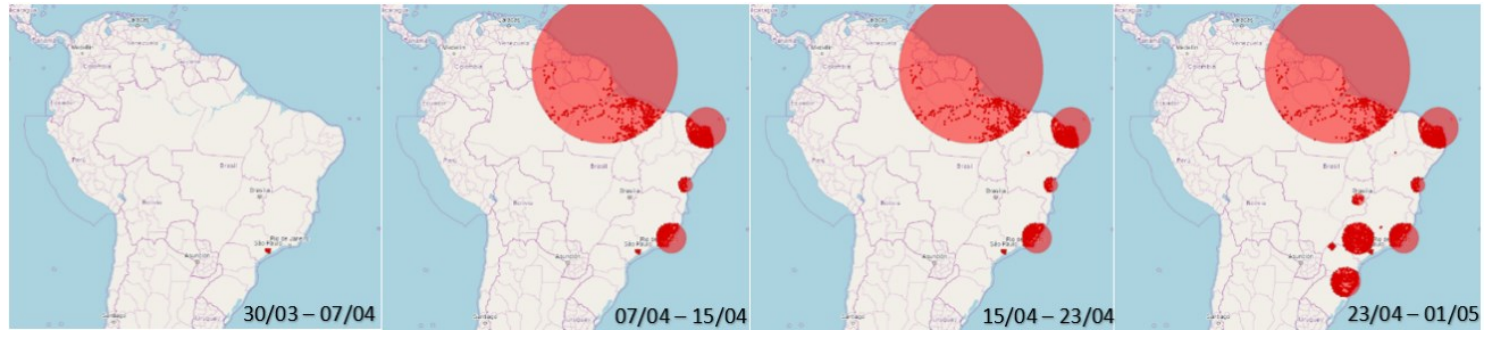

Fonte: https://espacourbanoesaude.iea.usp.br/monitora-clusters/.

Os agrupamentos ativos de óbitos, por sua vez, estão representados na Figura 3 . No período de 25/02 a 01/05 foram identificados 8 agrupamentos ativos. O mais importante contém 28 municípios, tem seu centro em Ferraz de Vasconcelos/SP, totaliza 1.827 óbitos e início em 06 de abril. No dia 12 de abril tiveram início dois agrupamentos: um no Nordeste, com centro em São Miguel dos Gostos/RN, envolvendo 500 municípios e 992 óbitos e outro no Sudeste, com centro em Serra/ES, envolvendo mais 6 municípios e 74 óbitos. Em 14 de abril surge um agrupamento em Duque de Caxias/RJ somando-se a 8 outros municípios, onde ocorreram 621 óbitos até o dia 1 de maio. No dia 22 de abril tiveram início mais 3 agrupamentos. O mais importante destes ocorreu na Região Norte, envolvendo 371 municípios, com centro em Vitória do Jari/PA e 664 óbitos. O segundo, no oeste do Estado de São Paulo e norte do Paraná, com centro em Taquarituba/SP, apresentou 203 óbitos. O município de Salvador/BA, constituiu um agrupamento ativo a partir desta data, quando, até 1 de maio tinham ocorrido 53 mortes. Outros 102 municípios constituem o agrupamento da Região Sul, centrado em Arvorezinha/RS, onde ocorreram 21 óbitos.

Figura 3 - Agrupamentos espaço-temporais de excesso de óbitos por COVID-19 ativos até o dia $01 / 05$

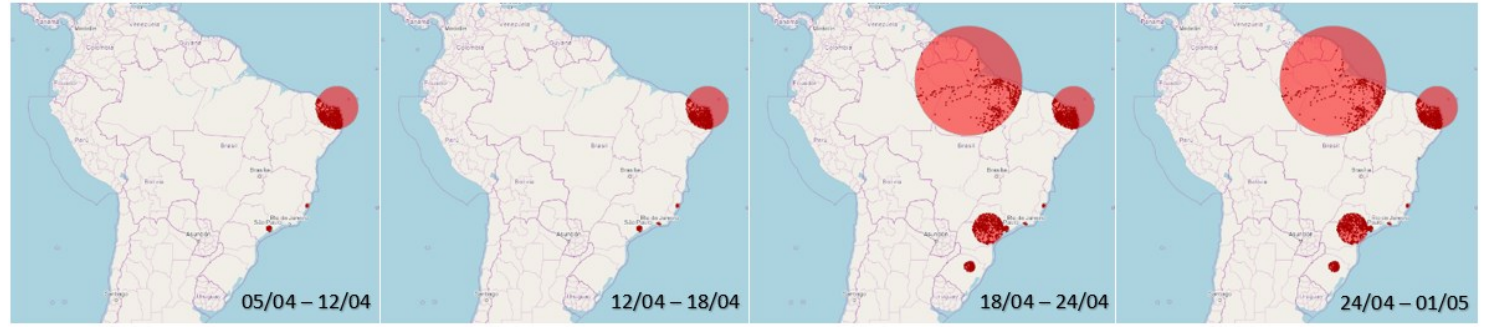

Fonte: https://espacourbanoesaude.iea.usp.br/monitora-clusters/. 


\section{CONSIDERAÇÕES FINAIS}

A identificação dos casos de COVID-19 no Brasil vem sendo marcada por um cenário crítico de subnotificação. A aplicação dos testes varia conforme a capacidade de enfrentamento dos estados e municípios à pandemia, o que é bastante diverso no contexto nacional. Nesse sentido, algumas unidades federativas têm mais casos porque aplicaram um maior número de testes. A subnotificação representa um importante desafio, de modo que o cenário apresentado possui limitações em retratar fidedignamente a complexidade do espalhamento espaço-temporal da doença. Contudo, a partir dos dados disponíveis, o reconhecimento e delimitação dos agrupamentos são um potente instrumento de alerta aos gestores. O confrontamento dos números de óbitos ocorridos nos meses de 2020 com os óbitos médios ocorridos mensalmente em anos anteriores, dão uma indicação do impacto da COVID19 e da sua subnotificação. No entanto, enquanto dados consistidos não estiverem disponíveis pelo Ministério da Saúde, com informações de sexo e idade, qualquer análise e projeção estatística subentende tal limitação.

É particularmente importante o acompanhamento do espalhamento da doença no Brasil, o quinto maior país do mundo em território e população e situado na periferia do capitalismo internacional. As cidades brasileiras são marcadas por enormes disparidades socioespaciais, com elementos claros de periferização oriundos do recorte de classes que marca o espaço nacional. $57,4 \%$ da população brasileira está concentrada em $5,8 \%$ dos municípios, de modo que cidades médias e grandes (aquelas com o mínimo de cem mil habitantes) no contexto brasileiro, mas não somente, apresentam maiores densidades demográficas, verticalização, redes de transporte capilarizadas e internalizadas, favelização, redes de água e esgoto ausentes ou precárias em parte das cidades, somando elementos que contribuem para o espalhamento da COVID-19.

A apresentação interativa espaço-temporal proporcionada pelo ArcGis online permite ver o surgimento dos agrupamentos e o quadro dos agrupamentos ativos até a data da atualização dos dados. Com o desenvolvimento da epidemia, alguns agrupamentos irão desaparecer e outros poderão surgir, o que torna esta ferramenta muito útil para o acompanhamento desta evolução para orientar as medidas apropriadas para suporte ao sistema de saúde e recomendações para o distanciamento social ou, até mesmo, lockdown (bloqueio total). Compreender a evolução da epidemia pode contribuir para o enfrentamento desta e das possíveis epidemias futuras.

\section{AGRADECIMENTO}

A Fabio Franco, médico infectologista do Hospital Universitário da Universidade de São Paulo, pela revisão e sugestões.

\section{REFERÊNCIAS}

ADHIKARI, S.P.; MENG, S.; WU, Y-J; MAO, Y-P. et al. Epidemiology, causes, clinical manifestation and diagnosis, prevention and control of coronavirus disease (COVID-19) during the early outbreak period: a scoping review. Infectious Diseases of Poverty, v. 9, n. 29, p. 1-12, 2020. https://doi.org/10.1186/s40249-020-00646-x

BRASIL IO. COVID-19. Disponível em: https://brasil.io/dataset/covid19/caso . Acesso em: 03 mai. 2020.

DESJARDINS, M. R.; HOHL, A.; DELMELLE, E. M. Rapid surveillance of COVID-19 in the United States using a prospective space-time scan statistic: Detecting and evaluating emerging clusters. Applied Geography, v. 118, p. 1-7, 2020. https://doi.org/10.1016/j.apgeog.2020.102202

EARLY ALERT. Coronavirus 2020 (dashboard). Disponível em: https://earlyalert.maps.arcgis.com/apps/opsdashboard/index.html\#/20bfbf89c8e74c0494c90b1ae0fa7b78. Acesso em: 28 abr. 2020.

GIULIANI, D.; DICKSON, M.M.; ESPA, G.; SANTI, F. Modelling and predicting the spatio-temporal spread of Coronavirus disease 2019 (COVID-19) in Italy. 2020. Disponível em: https://arxiv.org/pdf/2003.06664.pdf . Acesso em: 29 abr. 2020. https://doi.org/10.2139/ssrn.3559569

GULIYED, H. Determining the spatial effects of COVID-19 using the spatial panel data model. Spatial Statistics, v. 38, p. 1-10, 2020. https://doi.org/10.1016/i.spasta.2020.100443 
HAMID, S.; MIR, MY; ROHELA, GK. Novel coronavirus disease (COVID-19): a pandemic (epidemiology, pathogenesis and potential therapeutics). New Microbes and New Infections, v. 35, p. 1-10, 2020. https://doi.org/10.1016/i.nmni.2020.100679

HUANG, C.; WANG, Y.; LI, X.; REN, L. et al. Clinical features of patients infected with 2019 novel coronavirus in Wuhan, China. The Lancet, v. 395, p. 497-506, 2020. https://doi.org/10.1016/S0140$\underline{6736(20) 30183-5}$

INSTITUTO BRASILEIRO DE GEOGRAFIA E ESTATÍSTICA. Estimativas da População (TCU 2019). Disponível em: https://www.ibge.gov.br/estatisticas/sociais/populacao/9103-estimativas-depopulacao.html?=\&t=downloads. Acesso em: 09 abr. 2020.

INSTITUTO BRASILEIRO DE GEOGRAFIA E ESTATÍSTICA. Malhas municipais. Disponível em: ftp://geoftp.ibge.gov.br/organizacao_do_territorio/malhas_territoriais/malhas_municipais. Acesso em: 10 abr. 2020.

JOHNS HOPKINS UNIVERSITY, CSSE. Coronavirus COVID-19 Global Cases (dashboard). Disponível https://gisanddata.maps.arcgis.com/apps/opsdashboard/index.html\#/bda7594740fd40299423467b48e 9ecf6. Acesso em: 27 abr. 2020.

KAMEL BOULOS, M. N.; GERAGHTY, E. M. Geographical tracking and mapping of coronavirus disease COVID-19/severe acute respiratory syndrome coronavirus 2 (SARS-CoV-2) epidemic and associated events around the world: how 21st century GIS technologies are supporting the global fight against outbreaks and epidemics. International Journal of Health Geographics, v. 19, n. 8, p. 1-12, 2020. https://doi.org/10.1186/s12942-020-00202-8

KANG, D.; CHOI, H.; KIM, J-H; CHOI, J. Spatial epidemic dynamics of the COVID-19 outbreak in China. International Journal of Infectious Diseases, v. 94, p. 96-102, 2020. https://doi.org/10.1016/j.jijid.2020.03.076

KOST, G. J. Geospatial hotspots need point-of-care strategies to stop highly infectious outbreaks: ebola and coronavirus. Archives of Pathology \& Laboratory Medicine, p. 1-64, 2020. https://doi.org/10.5858/arpa.2020-0172-RA

KULLDORFF, M. Prospective time periodic geographical disease surveillance using a scan statistic. Journal of the Royal Statistical Society: Series A, v. 164(1), p. 61-72, 2001. https://doi.org/10.1111/1467-985X.00186

KULLDORFF, M. Information Management Services Inc. SaTScanTM v9.6: Software for the spatial and space-time scan statistics. 2018. Disponível para download e instalação em: http://www.satscan.org

LAKHANI, A. Commentary Introducing the Percent, Number, Availability, and Capacity [PNAC] Spatial Approach to Identify Priority Rural Areas Requiring Targeted Health Support in Light of COVID-19: A Commentary and Application. The Journal of Rural Health, p. 1-7, 2020a. https://doi.org/10.1111/jrh.12436

LAKHANI, A. Which Melbourne metropolitan areas are vulnerable to COVID-19 based on age, disability and access to health services? Using spatial analysis to identify service gaps and inform delivery. Journal of Pain and Symptom Management. 2020b. https://doi.org/10.1016/i.jpainsymman.2020.03.041

LIU, T.; HU, J.; XIAO, J.; HE, G.; KANG, M.; RONG, Z. et al. Time-varying transmission dynamics of Novel Coronavirus Pneumonia in China. 2020. https://doi.org/10.1101/2020.01.25.919787

LU, H.; STRATTON, C. W.; TANG, Y-W. Outbreak of pneumonia of unknown etiology in Wuhan, China: the mystery and the miracle. Journal of Medical Virology, v. 92, p. 401-402, 2020. https://doi.org/10.1002/jmv.25678

WILSON, J. G.; BALLOU, J.; YAN, C.; FISHER-HOCH, S.P.; REININGER, B.; GAY, J. et al. Utilizing spatiotemporal analysis of influenza-like illness and rapid tests to focus swine-origin influenza virus $\begin{array}{lllllll}\text { intervention. Health \& Place. V. } 16, \quad \text { n. } 6, & \text { p. } 1230-1239, & 2010 .\end{array}$ https://doi.org/10.1016/i.healthplace.2010.08.010 
WORLD HEALTH ORGANIZATION. Novel coronavirus (COVID-19) situation (dashboard). Disponível em: http://healthcybermap.org/WHO_COVID19/. Acesso em: 28 abr. 2020.

YANG, W.; DENG, M.; LI, C.; HUANG, J. Spatio-Temporal Patterns of the 2019-nCoV Epidemic at the County Level in Hubei Province, China. International Journal of Environmental Research and Public Health, v. 17, p. 1-11, 2020. https://doi.org/10.3390/ijerph17072563

ZHOU, C.; SU, F.; PEI, T.; ZHANG, An.; DU, Y.; LUO, B. et al. COVID-19: Challenges to GIS with Big Data. Geography Sustainability, v. 1, p. 77-87, 2020. https://doi.org/10.1016/j.geosus.2020.03.005 